\title{
Centrosomal protein FOR20 is essential for S-phase progression by recruiting PIk1 to centrosomes
}

\author{
Minhong Shen ${ }^{1,2}$, Yuqi Cai ${ }^{1,2}$, Yuehong Yang ${ }^{1,2}$, Xiaoyi Yan ${ }^{1,2}$, Xiaoqi Liu ${ }^{3}$, Tianhua Zhou ${ }^{1,2}$ \\ ${ }^{I}$ Department of Cell Biology and Program in Molecular Cell Biology, Zhejiang University School of Medicine, 866 Yuhangtang \\ Road, Hangzhou, Zhejiang 310058, China; ${ }^{2}$ Collaborative Innovation Center for Diagnosis and Treatment of Infectious Diseases, \\ Hangzhou, Zhejiang 310003, China, ${ }^{3}$ Department of Biochemistry, Purdue University, West Lafayette, IN 47907, USA
}

Centrosomes are required for efficient cell cycle progression mainly by orchestrating microtubule dynamics and facilitating G1/S and G2/M transitions. However, the role of centrosomes in S-phase progression is largely unknown. Here, we report that depletion of FOR20 (FOP-related protein of $20 \mathrm{kDa}$ ), a conserved centrosomal protein, inhibits S-phase progression and prevents targeting of Plk1 (polo-like kinase 1) to centrosomes, where FOR20 interacts with Plk1. Ablation of Plk1 also significantly induces S-phase defects, which are reversed by ectopic expression of PIk1, even a kinase-dead mutant, but not a mutant that fails to localize to centrosomes. Exogenous expression of centrosome-tethered Plk1, but not wild-type Plk1, overrides FOR20 depletion-induced S-phase defects independently of its kinase activity. Thus, these data indicate that recruitment of Plk1 to centrosomes by FOR20 may act as a signal to license efficient progression of S-phase. This represents a hitherto uncharacterized role of centrosomes in cell cycle regulation.

Keywords: cell cycle; FOR20; Plk1; S-phase; DNA replication

Cell Research (2013) 23:1284-1295. doi:10.1038/cr.2013.127; published online 10 September 2013

\section{Introduction}

In most animal cells, centrosomes serve as a scaffold for anchoring hundreds of regulatory proteins that participate in cell locomotion, division and sensory reception mainly by orchestrating microtubule dynamics [1-3]. Recently, emerging data suggest that centrosomes are essential for several cell cycle transitions, including the G1/ $\mathrm{S}$ and $\mathrm{G} 2 / \mathrm{M}$ transitions $[2,3]$. Removal of centrosomes by microsurgery either in interphase cells or in prophase cells ultimately results in failure of these cells to enter into subsequent S-phase, as does depletion of some centrosome-associated proteins by RNA interference (RNAi) [3-5], implying a possible centrosomal checkpoint at the G1/S boundary. This hypothesis is further supported by the fact that either centrosomal cyclin $\mathrm{E}$ or $\mathrm{A}$ is essential for S-phase entry [6-8]. Some key mitotic regulators, such as cyclin-dependent kinase 1 (CDK1), Aurora A and

Correspondence: Tianhua Zhou

Tel: +86 571 88208258; Fax: +86 57188208069

E-mail: tzhou@zju.edu.cn

Received 2 May 2013; revised 13 July 2013; accepted 16 July 2013; published online 10 September 2013 polo-like kinase 1 (Plk1), are recruited to centrosomes at the G2/M transition, where they are fully activated, presumably through centrosome-localized phosphatase Cdc25B $[2,6,9,10]$, suggesting an important role of centrosomes in mitotic entry. However, little is known about the role of centrosomes during S-phase progression.

Recently, an evolutionally conserved protein, FOR20 (FOP-related protein of $20 \mathrm{kDa}$ ), has been identified; it is associated with centrosomes and may be involved in ciliogenesis $[11,12]$. Here, we find that FOR20 is essential not only for S-phase progression, but also for recruiting Plk1 to centrosomes. Further data show that targeting Plk1 to centrosomes is crucial for DNA replication independently of its kinase activity, suggesting that the recruitment of Plk1 to centrosomes by FOR20 may act as an "intra-S-phase" signal to license/permit efficient progression of S-phase.

\section{Results}

The role of FOR20 in S-phase progression

To explore the role of centrosomal protein FOR20 in cell cycle regulation, we raised a rabbit polyclonal an- 
tibody against purified FOR20 protein (Supplementary information Data S1 and Figure S1A). This antibody specifically recognized endogenous FOR20 in mammalian cells (Supplementary information, Data S1 and Figure S1B). Immunofluorescence staining showed that FOR20 colocalized with the centrosomal proteins $\gamma$-tubulin and centrin-2 (Supplementary information, Figure S1C), which was validated by co-purification of FOR20 and $\gamma$-tubulin in the centrosomal fraction by sucrose gradient centrifugation (Supplementary information, Figure S1D). These results are consistent with the previous studies $[11,12]$. Next, we used vector-based RNAi to deplete endogenous FOR20 in mammalian cells. Western blotting and immunofluorescence analyses showed that FOR20 was greatly reduced and clearly absent from centrosomes in FOR20-depleted cells (Figure 1A and 1B). In addition, phase-contrast microscopy, cell count and MTT (3-[4, 5-dimethylthiazol-2-yl]-2, 5-diphenyl-tetrazoliumbromide) assays revealed that depletion of FOR20 significantly inhibited cell proliferation (Supplementary information, Data S1 and Figure S2A-S2C).
To determine how FOR20 influences cell proliferation, we employed fluorescence-activated cell sorter (FACS) analysis and found that FOR20 ablation induced obvious defects in S-phase progression, which were reversed by ectopic expression of RNAi-resistant FOR20 (Figure 1C and 1D). This result was confirmed by FACS analysis with cells depleted of FOR20 by RNAi after double-thymidine block and release (Supplementary information, Figure S2D). To validate the role of FOR20 during Sphase, we used BrdU (5'-bromo-2'-deoxyuridine) to label cells in S-phase and found that FOR20 depletion significantly increased the population of cells in S-phase (Figure $1 \mathrm{E}$ and $1 \mathrm{~F})$. BrdU incorporation after double-thymidine block synchronization and release further confirmed the important role of FOR20 in S-phase progression (Supplementary information, Figure S2E). To eliminate the potential off-targeting effects, we employed another vectorbased RNAi that targets the previously described region [12] and found that depletion of endogenous FOR20 indeed induced significant defects in S-phase (Supplementary information, Data S1 and Figure S3).
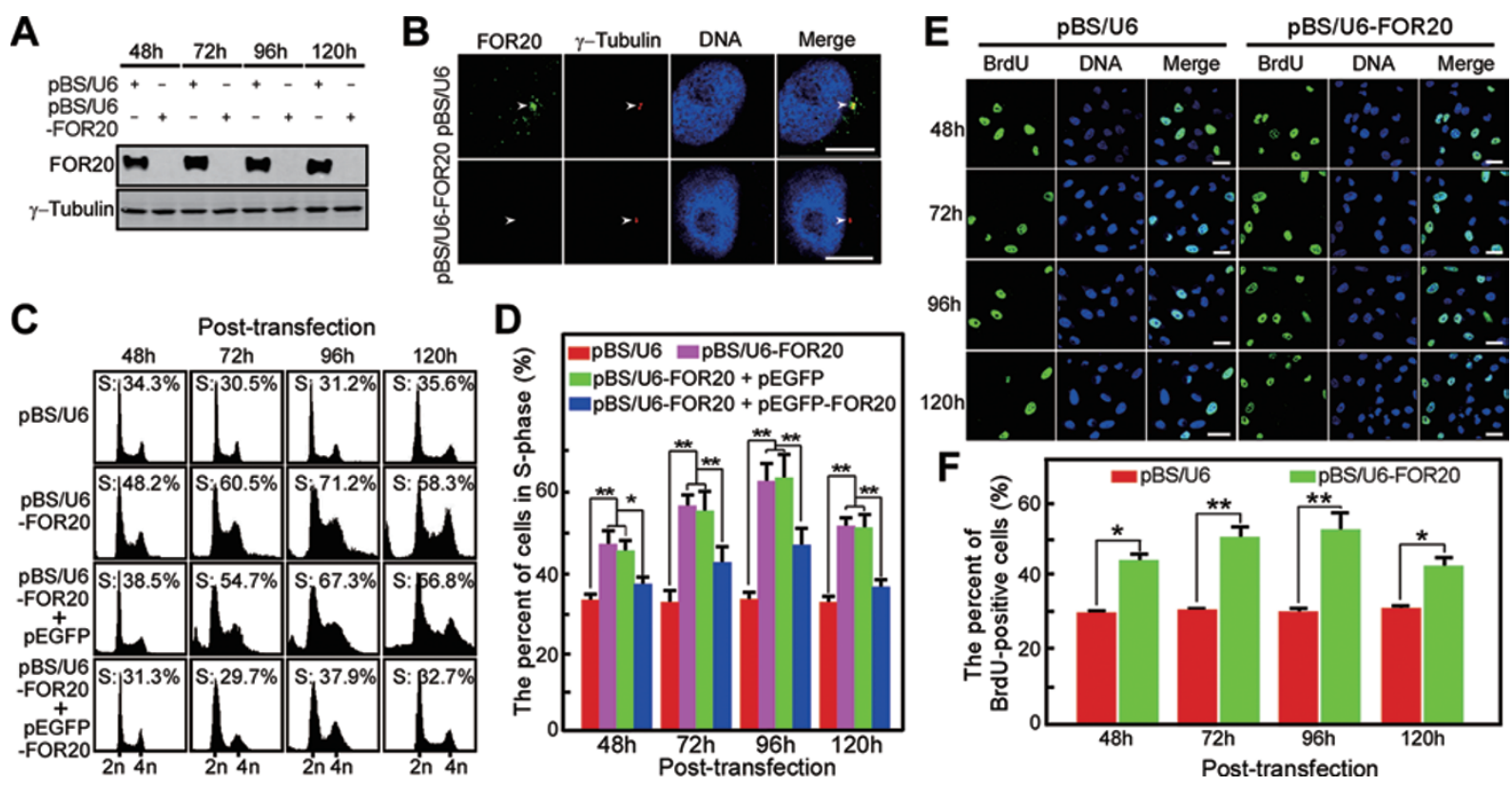

Figure 1 Depletion of FOR20 inhibits S-phase progression. (A) HeLa cells were transfected with either pBS/U6 or pBS/U6FOR20 for different times. Centrosomes were purified by sucrose gradient ultracentrifugation and analyzed by western blotting with the antibodies against FOR20 or $\gamma$-tubulin. (B) The cells transfected with either pBS/U6 or pBS/U6-FOR20 for $96 \mathrm{~h}$ were subjected to immunofluorescence staining with anti-FOR20 and $\gamma$-tubulin antibodies. DNA was stained with DAPI. Scale bar, $10 \mu \mathrm{m}$. (C, D) The cells transfected with the indicated plasmids for different times were analyzed by FACS. The vector pEGFP-FOR20 is resistant to FOR20 RNAi. The percent of cells in S-phase is shown as the mean of three independent experiments $\pm \mathrm{SD}\left({ }^{*} P<0.05,{ }^{* *} P<0.01, n>300\right)$. (E, F) Cells transfected with either pBS/U6 or pBS/U6-FOR20 for various times were incubated with $10 \mu \mathrm{M}$ BrdU for another $20 \mathrm{~min}$ and subjected to immunofluorescence analysis with the anti-BrdU antibody and DAPI. Scale bar, $20 \mu \mathrm{m}$. The percent of BrdU-positive cells is expressed as the mean of three independent experiments $\pm \mathrm{SD}\left({ }^{*} P<0.05 ;{ }^{* *} P<0.01, n>300\right)$. 
FOR20 is required for targeting Plk1 to centrosomes

To examine the mechanism underlying the function of centrosomal FOR20 in S-phase progression, we tested the proper localization of centrosome-associated proteins, including CDK2, centrin-2, cyclin A, cyclin $\mathrm{E}$, dynein, p150 dynactin and Plk1, in cells depleted of FOR20. Immunofluorescence analysis showed that only Plk1, but not the other proteins, was absent from centrosomes in FOR20-depleted cells (Figure 2A and 2B). This is supported by the data that Plk1 did not co-purify with $\gamma$-tubulin during sucrose gradient centrifugation of FOR20-depleted cell extracts (Figure 2C). Interestingly, in cells depleted of Plk1, FOR20 was still colocalized with $\gamma$-tubulin (Supplementary information, Figure S4). These results suggest that FOR20 may be involved in the recruitment of Plk1 to centrosomes, but not vice versa.

Previous studies demonstrated that Plk1 can be detected at centrosomes in G2 phase [13, 14], yet much less is known about whether Plk1 is targeted to centrosomes during S-phase. Here, our data reveal that Plk1 was localized to centrosomes in S-phase cells labeled by either BrdU or proliferating cell nuclear antigen (PCNA) (Supplementary information, Figure S5A and S5B), indicating that Plk1 may associate with centrosomes during S-phase. To further test this assumption, we employed the double thymidine block method to synchronize HeLa cells and observed that Plk1 did colocalize with $\gamma$-tubulin during S-phase (Supplementary information, Figure S5CS5E).

To further investigate the dynamic localization of centrosomal Plk1, human glioblastoma T98G cells were arrested at G0 phase by deprivation of serum for $72 \mathrm{~h}$ and released into $20 \%$ serum for cell cycle reentry as previously described [15] (Supplementary information, Data S1). FACS profiles, western blotting and immunofluorescence microscopy showed that Plk1 began to express and associate with both $\gamma$-tubulin and FOR2 20 at the $\mathrm{G} 1 / \mathrm{S}$ transition ( $16 \mathrm{~h}$ post release), whereas FOR20 was persistently localized to centrosomes throughout cell cycle progression (Supplementary information, Figure S6). Taken together, our data indicate that FOR20 may be involved in targeting Plk1 to centrosomes at the G1/S transition.

\section{FOR20 interacts with Plk1 at centrosomes}

To study how FOR20 influences the recruitment of Plk1 to centrosomes, we examined the possible interaction between FOR20 and Plk1. GST pull-down and immunoprecipitation assays showed that FOR20 was able to bind Plk1 in vitro and form a complex with Plk1 in mammalian cells (Figure 3A-3C and Supplementary information, Figure S7A). As FOR20 associates with cen-
A

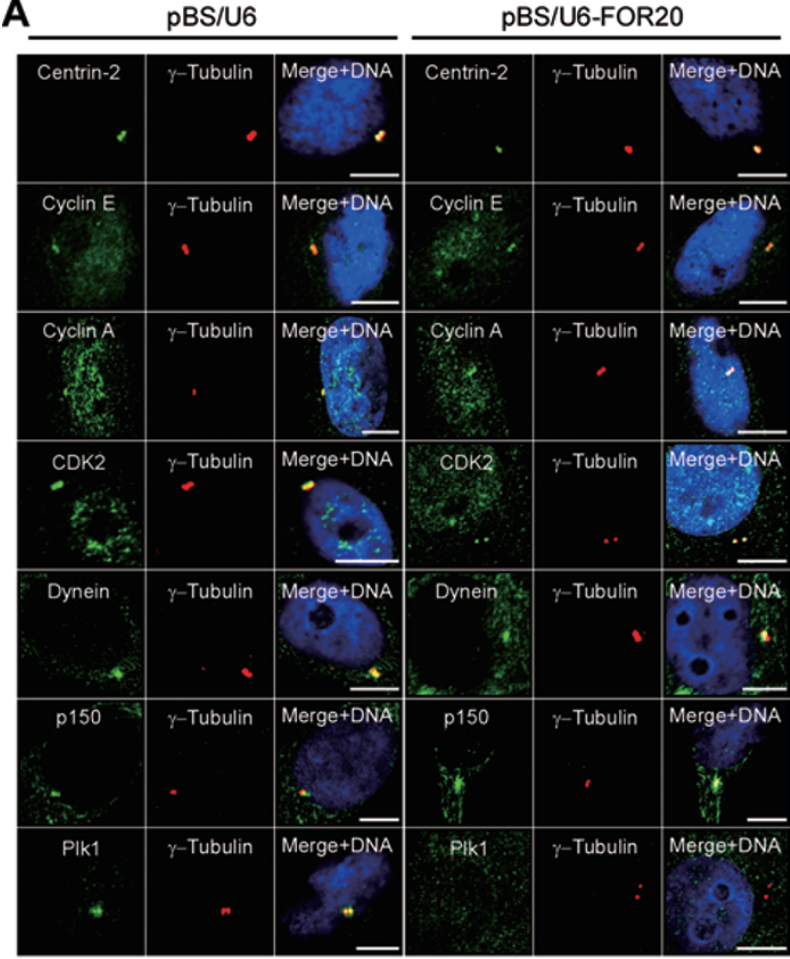

B

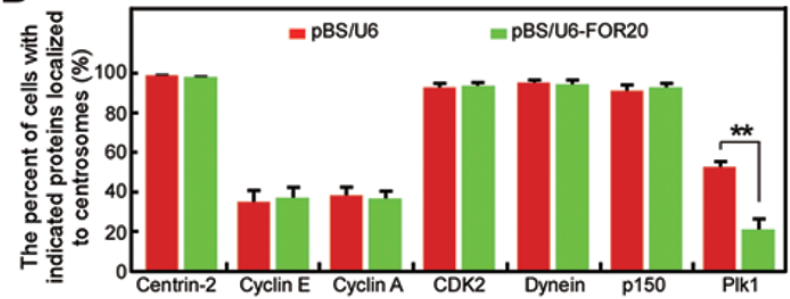

C
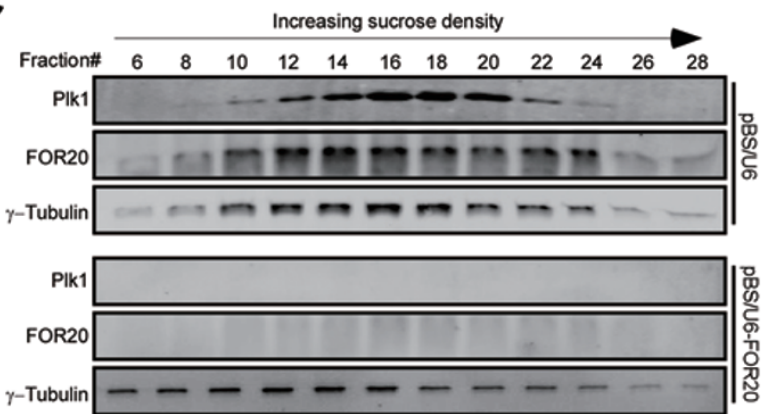

Figure 2 FOR20 is required for the centrosomal localization of Plk1. HeLa cells transfected with either pBS/U6 or pBS/U6FOR20 for $96 \mathrm{~h}$ were subjected to multiple analyses. (A) The cells were processed for immunofluorescence microscopy with the indicated antibodies. DNA was visualized by DAPI. Scale bar, $10 \mu \mathrm{m}$. (B) The percent of cells with the indicated proteins localized to centrosomes is shown as the mean of three independent experiments \pm SD $\left({ }^{* *} P<0.01, n>300\right)$. (C) The centrosomal fractions of HeLa cells were purified by sucrose gradient ultracentrifugation and then immunoblotted with the indicated antibodies. 
A

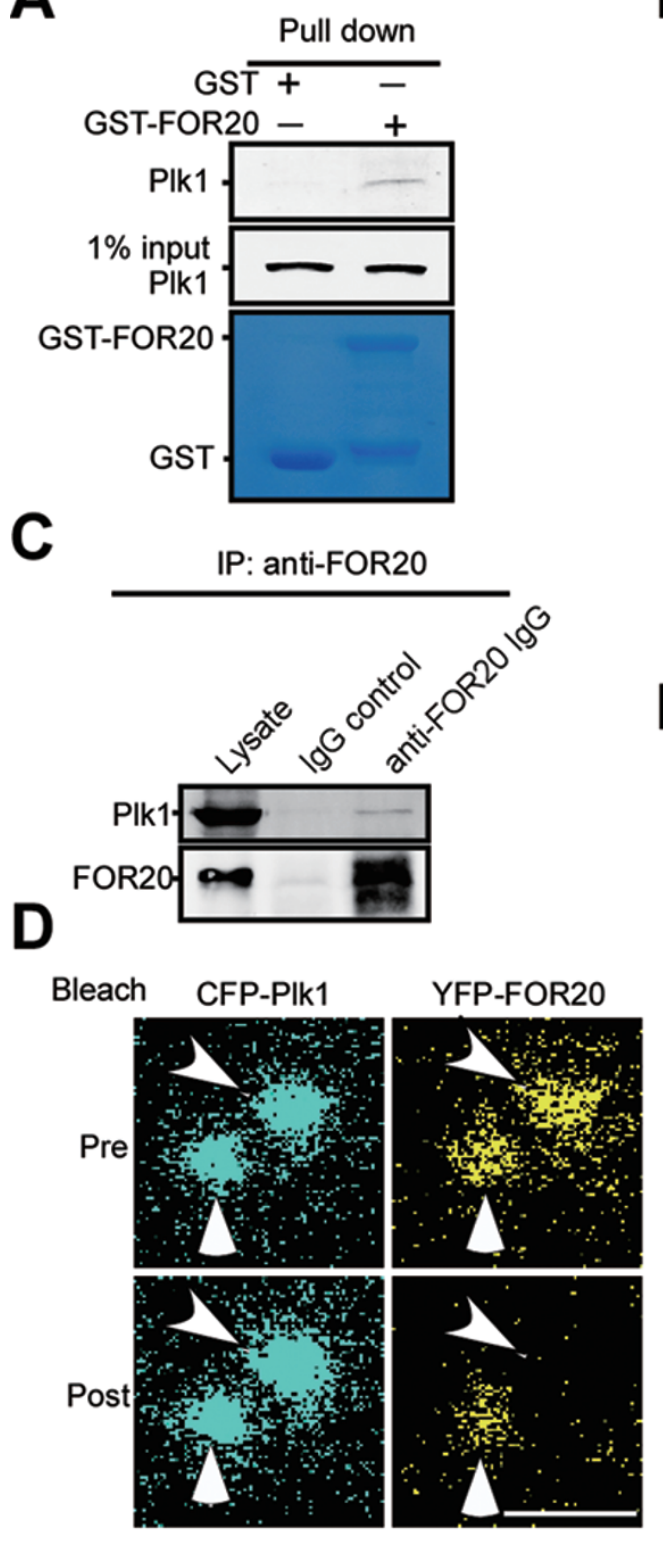

B
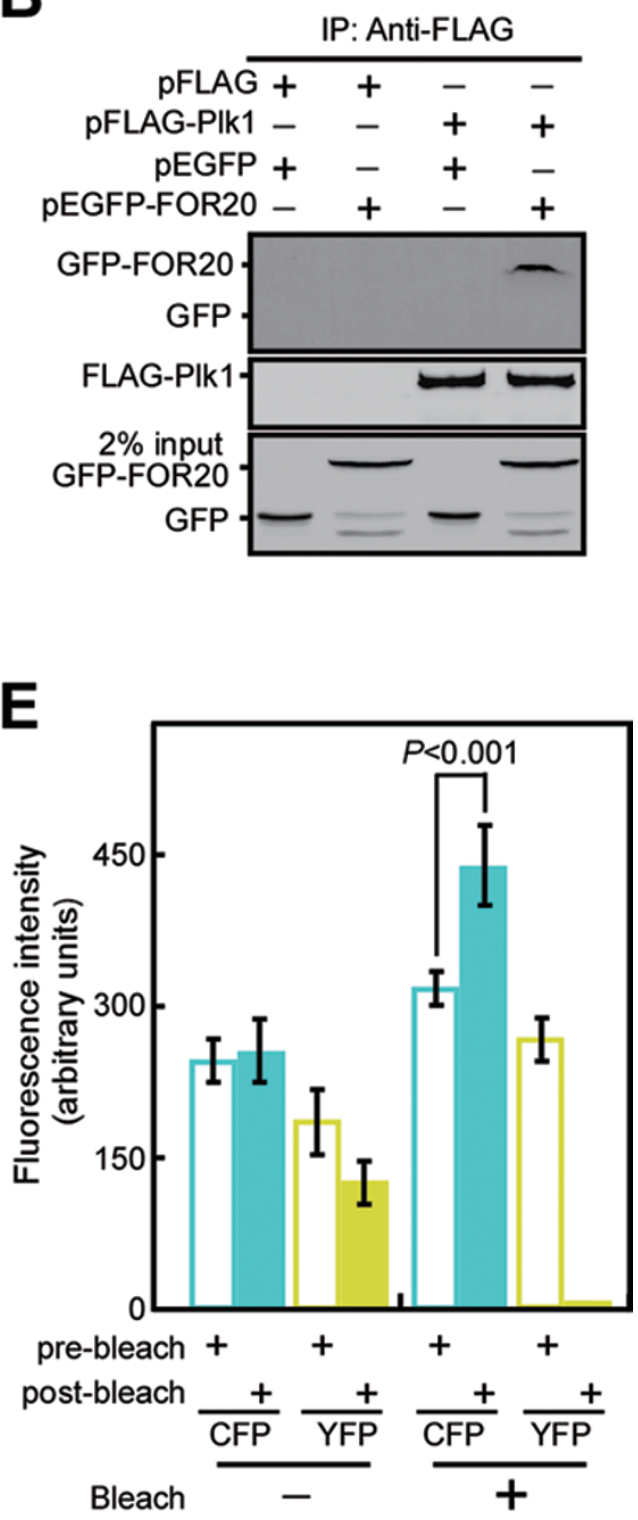

Figure 3 FOR20 interacts with Plk1 at centrosomes. (A) Bacterially expressed GST-FOR20 was incubated with lysates of HeLa cells. Endogenous PIk1 that bound to GST-FOR20 was detected by immunoblot analysis with anti-PIk1 antibody. Purified GST and GST-FOR20 proteins used in this pull-down assay were resolved by SDS-PAGE and subjected to Coomassie brilliant blue staining. (B) HeLa cells transfected with the indicated vectors for $24 \mathrm{~h}$ were subjected to coimmunoprecipitation with anti-FLAG antibody-coupled beads. Immunoblot analyses were performed with the antibodies as shown. (C) Lysates of HeLa cells were immunoprecipitated with either anti-rabbit IgG or anti-FOR20 antibody, and the immunoprecipitated proteins were analyzed by western blotting with the indicated antibodies. (D, E) HeLa cells expressing CFP-PIk1 and YFP-FOR20 were subjected to FRET analysis by acceptor photobleaching. A representative image from $>15$ experiments is shown. Only one (arrowhead) of the two centrosomes was photobleached. "Pre" and "Post" stand for before and after photobleaching, respectively. Scale bar, $2 \mu \mathrm{m}$. Fluorescence intensities of pre (open bar) and post (filled bar) are shown as the mean of three independent experiments \pm SD.

trosomes throughout the cell cycle, and Plk1 begins to be recruited to centrosomes at the G1/S transition, we tested whether the endogenous interaction between FOR20 and
Plk1 is specific to certain phases of the cell cycle. The results of co-immunoprecipitation after cell synchronization and release revealed that endogenous FOR20 inter- 
acted with Plk1 in later G1, S, G2 or M phase (Supplementary information, Figure S7B and S7C), suggesting that the interaction of FOR20 and Plk1 is not restricted to S-phase.

To investigate whether FOR20 associates with Plk1 at centrosomes, we applied fluorescence resonance energy transfer (FRET) analysis. HeLa cells transfected with both pECFP-Plk1 and pEYFP-FOR20 vectors were fixed and subjected to acceptor photobleaching to calculate FRET efficiency. After YFP bleaching, the CFP fluorescence intensity of the bleached centrosome significantly increased compared with that of the non-bleached centrosome (Figure 3D and 3E). Thus, these results imply that FOR20 may interact with Plk1 at centrosomes.

\section{Centrosomal Plk1 is essential for S-Phase progression}

Since Plk1 appears to be expressed during S-phase, we tested whether Plk1 contributes to S-phase progression. Cells depleted of Plk1 were treated with thymidine, and subjected to western blotting and FACS analysis (Figure 4A and 4B). The data show that depletion of Plk1 obviously inhibited S-phase progression (Figure 4B, lanes 1 and 2), which was supported by our results from BrdU incorporation after thymidine block and release (Supplementary information, Data S1 and Figure S8A) and the previously described data [16-18].
As Plk1 associates with centrosomes during S-phase and plays a role in S-phase progression, we tried to determine whether the recruitment of Plk1 to centrosomes is required for S-phase progression. Previous reports demonstrated that the conserved residues (W414, V415 and L427) of Plk1 are required for its centrosomal localization $[19,20]$. We first constructed two Plk1 mutants, pEGFP-Plk1-FA (Plk1-W414F/L427A) and pEGFP-Plk1-FAA (Plk1-W414F/V415A/L427A). Immunofluorescence data confirmed that most GFP-Plk1FAA were unable to associate with centrosomes (Figure 4D and 4E). Next, we examined the effect of these Plk1 mutants on the S-phase defects caused by the depletion of Plk1. Ectopic expression of GFP-Plk1-WT or GFPPlk1-FA, but not GFP-P1k1-FAA that fails to localize to centrosomes, significantly reversed Plk1 depletioninduced S-phase defects (Figures 4B, lanes 3 to 5, and Supplementary information, Figure S8A). Unexpectedly, the kinase-dead mutant of Plk1 (GFP-Plk1-K82M) was also effective in this regard (Figure $4 \mathrm{C}$ and Supplementary information, Figure S8B), indicating that the kinase activity of Plk1 is dispensable for its roles in S-phase progression. Furthermore, neither the $\mathrm{N}$ terminus (GFPPlk1-N) nor C terminus (GFP-Plk1-C) of Plk1 efficiently reversed the S-phase defects induced by Plk1 depletion as GFP-Plk1-K82M did (Figure 4C and Supplementary
A

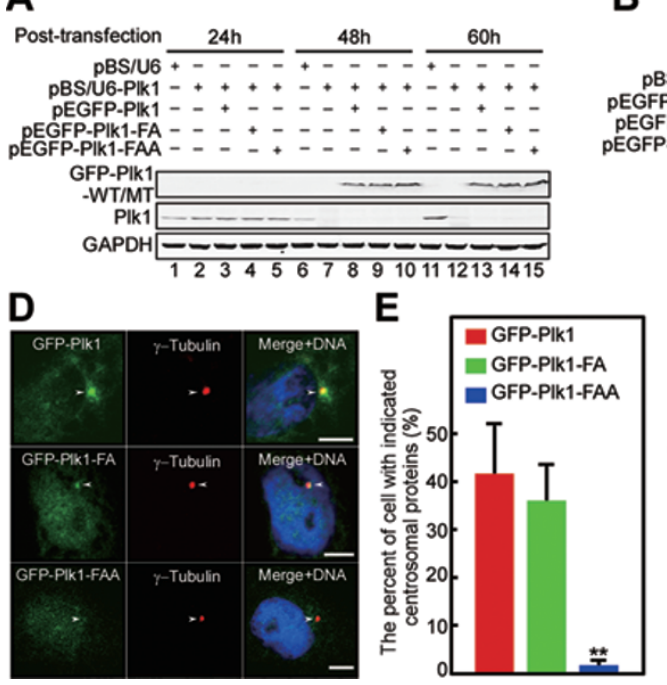

B

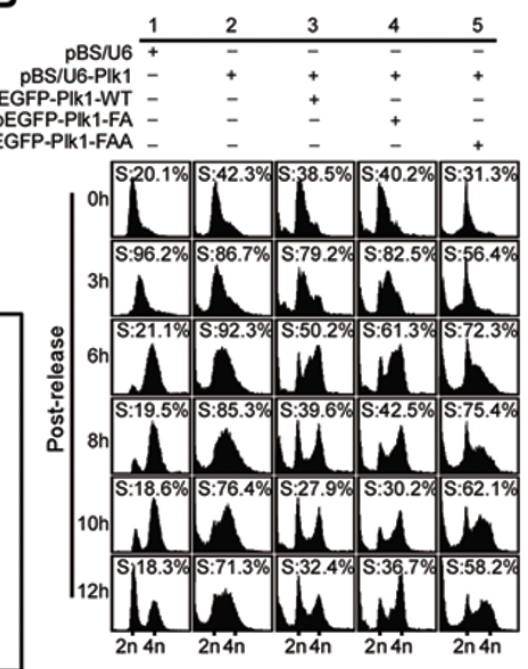

C

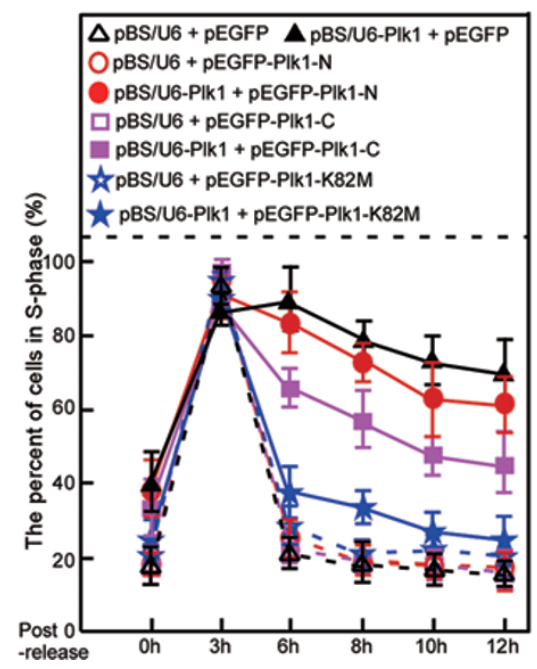

Figure 4 Plk1 recruitment to centrosomes is crucial for S-phase progression. (A-E) HeLa cells treated with either pBS/U6 or pBS/U6-Plk1 for $24 \mathrm{~h}$ were transfected with the indicated vectors and blocked with thymidine $(2.5 \mathrm{mM}, 24 \mathrm{~h})$. These cells were released from the thymidine block and subjected to western blot $(\mathbf{A})$ and FACS analysis at the indicated times (B, $\mathbf{C})$. The percent of cells in S-phase is shown as the mean of three independent experiments \pm SD (C). (D) HeLa cells transfected with pEGFP-PIk1-WT, pEGFP-PIk1-FA or pEGFP-PIk1-FAA for $24 \mathrm{~h}$ were fixed and stained with anti- $\gamma$-tubulin antibody (red). DNA was visualized by DAPI (blue). Scale bar, $10 \mu \mathrm{m}$. (E) The percent of cells with either centrosomal GFP-PIk1, GFP-PIk1FA or GFP-PIk1-FAA is shown as the mean of three independent experiments \pm SD $\left({ }^{* \star} P<0.01, n>300\right)$. 
information, Figure S8), suggesting that the structural integrity of Plk1 may be crucial for S-phase progression in this context.

To determine whether the role of centrosomal Plk1 in S-phase progression is relatively specific, we tested the effect of centrosomal localization of two other mitotic regulators, CDK1 and Aurora A kinase, on S-phase progression. The data show that depletion of either CDK1 or Aurora A kinase did not affect Plk1 recruitment to centrosomes and S-phase progression (Figure 5).

\section{FOR20 plays a role in $S$-Phase progression by recruiting Plk1 to centrosomes}

Considering that FOR20 is essential for both targeting Plk1 to centrosomes and S-phase progression, and that centrosomal Plk1 is indispensable for S-phase progression, we reasoned that FOR20 depletion-induced S-phase defects may be attributed to delocalization of Plk1 from centrosomes. To test this hypothesis, we investigated whether forced Plk1 localization to centrosomes could rescue the S-phase defects induced by FOR20 depletion. We fused GFP-Plk1 with a centrosomal-targeting domain of AKAP450 (A-kinase anchoring protein 450) to generate a Plk1 fusion protein that preferentially associates with centrosomes [21]. The resulting GFPPlk1-AKAP, but not GFP-Plk1, was robustly localized to centrosomes in cells depleted of FOR20 (Figure 6A and $6 \mathrm{~B}$ ). Transfection of pEGFP-P1k1-AKAP, but not pEGFP-Plk1 or pEGFP-AKAP, was able to reverse the S-phase defects in FOR20-depleted cells (Figure 6C and Supplementary information, Figure S9A). Furthermore, forced centrosomal targeting of kinase-dead Plk1 (GFPPlk1-K82M-AKAP), polo-box domain (PBD)-deficient Plk1 (GFP-Plk1-FAA-AKAP), or even the kinase-dead, PBD-deficient Plk1 (GFP-Plk1-K82M-FAA-AKAP), but neither $\mathrm{N}$ terminus (GFP-Plk1-N-AKAP) nor C terminus (GFP-Plk1-C-AKAP) of Plk1, significantly reversed FOR20 depletion-induced S-phase defects (Figure 6D, $6 \mathrm{E}$ and Supplementary information, Figure S9B-S9D). Therefore, these results suggest that the recruitment of Plk1 to centrosomes by FOR20 is crucial for S-phase progression (Supplementary information, Figure S10).

\section{Discussion}

Centrosome duplication and DNA replication are tightly coordinated so that both occur once and only once per cell cycle. Previous studies have implied an important role for centrosomes in the G1/S and G2/M transitions [2, 3]. Recent reports show that centrosomal localization of either cyclin $\mathrm{E}$ or $\mathrm{A}$ is required for the initiation of DNA replication in the nucleus, while components of the DNA pre-replicative complex, including origin recognition complex 1 (ORC1) and minichromosome maintenance 5 (MCM5), are recruited to centrosomes and play a critical role in preventing centrosome reduplication $[7,8,22$, $23]$. These studies indicate that centrosomes are essential for entry into S-phase. However, little is known about the role of centrosomes during S-phase progression. Here, we provide evidence for the first time that targeting Plk1 to centrosomes promotes DNA replication. Depletion of Plk1 significantly inhibits S-phase progression, which is reversed by exogenous expression of Plk1, but not the Plk1 mutant that fails to localize to centrosomes (Figure 4 and Supplementary information, Figure S8). Ectopic expression of centrosome-tethered Plk1, but not wildtype Plk1, overrides the inhibition of DNA replication induced by depletion of FOR20 (Figure 6 and Supplementary information, Figure S9). Thus, these data suggest that recruitment of Plk1 to centrosomes by FOR20 may act as a signal to license effective progression of Sphase. This represents a previously undescribed role of centrosomes in cell cycle regulation.

Accumulating data suggest that there may be communications between centrosomes and the nucleus at Sphase entry [24]. Overexpression of the centrosomal localization signal of cyclin E prevents endogenous cyclin $\mathrm{E}$ from localizing to centrosomes, inhibits the initiation of DNA synthesis, and reduces the loading of initiation factor $\mathrm{Cdc} 45$ to chromatin [6-8, 24], indicating that a signal generated from centrosomes may be transmitted to the DNA replication complex in chromatin. Theoretically, there should be mutual communications between centrosomes and the nucleus to coordinate both centrosome and DNA events throughout the entire cell cycle. In this context, Plk1 appears to be involved in communications between centrosomes and the nucleus during Sphase progression.

It has been reported that the role of centrosomal cyclin $\mathrm{E}$ in initiation of DNA synthesis is dependent on an active CDK2-cyclin E complex [6]. However, our results show that centrosomal Plk1 facilitates DNA replication in a kinase activity-independent manner (Figures 4 and 6 ), suggesting a previously uncharacterized function of Plk1 during DNA replication. One possible interpretation is that centrosomal Plk1 may serve as a scaffold for recruiting DNA replication factors to centrosomes, which then generates signals that influence S-phase progression. Several DNA replication factors, including geminin, ORC and MCM proteins, localize to centrosomes and play crucial roles in the centrosome cycle [24]. Some ORC and MCM proteins not only colocalize with Plk1 at centrosomes, but also interact with Plk1 [2426]. Moreover, our results reveal that neither the PBD 
A

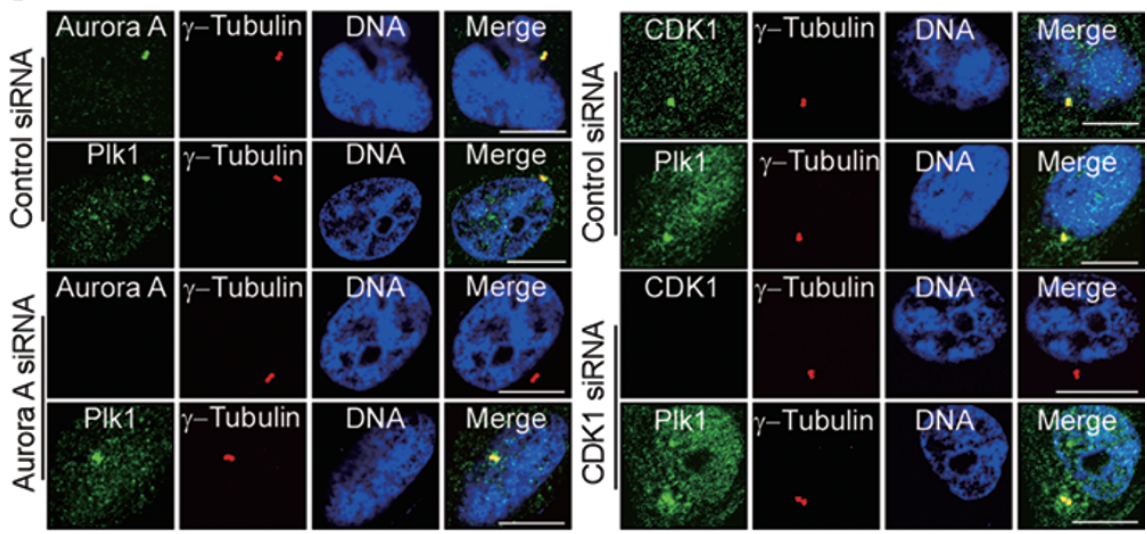

B

Post-release
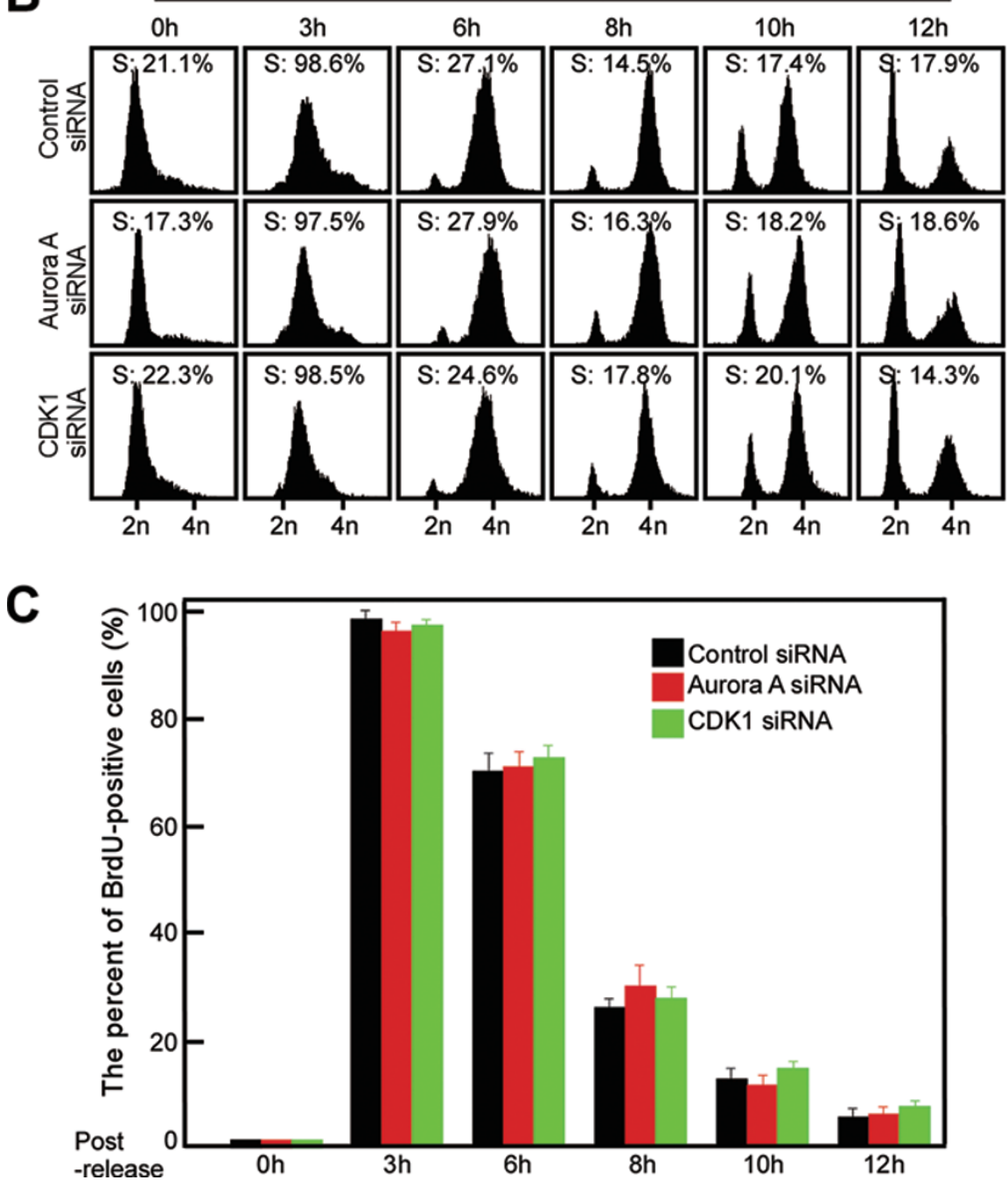

Figure 5 The role of centrosome-associated proteins Aurora A and CDK1 in S-phase progression. (A) HeLa cells were transfected with the indicated siRNAs for $36 \mathrm{~h}$ and subjected to immunofluorescence with the antibodies shown. DNA was stained with DAPI. Scale bar, $10 \mu \mathrm{m}$. (B, C) The cells transfected with the designated siRNAs for $12 \mathrm{~h}$ were synchronized with thymidine block for another $24 \mathrm{~h}$, and then subjected to FACS analysis (B) and immunofluorescence staining for BrdU incorporation $(\mathbf{C})$ at the indicated times after release. The percent of BrdU-positive cells is shown as the mean of three independent experiments $\pm \mathrm{SD}$. 
A

pBS/U6

pBS/U6-FOR20

\begin{tabular}{|c|c|c|c|c|c|c|c|}
\hline$\gamma$-Tubulin & $\begin{array}{c}\text { FOR20 } \\
\\
*\end{array}$ & $\begin{array}{c}\text { GFP.PIk1 } \\
\\
\alpha\end{array}$ & $\begin{array}{c}\text { Merge } \\
\boldsymbol{w}^{\prime}\end{array}$ & $\gamma$-Tubulin & FOR20 & GFP-PIk1 & Merge \\
\hline$\gamma$-Tubulin & FOR20 & $\begin{array}{c}\text { GFP-PIk1-AKAP } \\
-\end{array}$ & Merge & $\gamma$-Tubulin & FOR20 & GFP-PIk1-AKAP & Merge \\
\hline
\end{tabular}

B

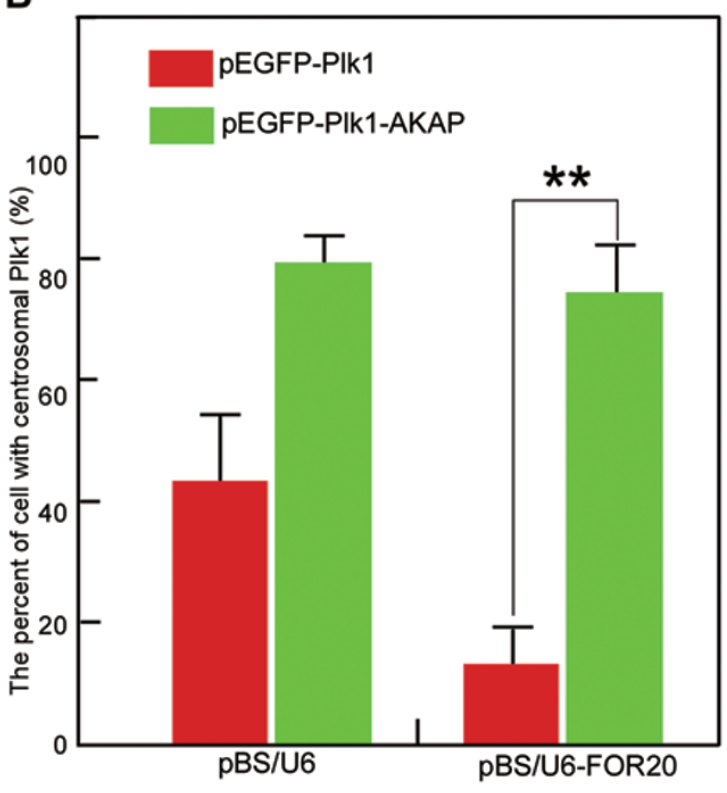

C

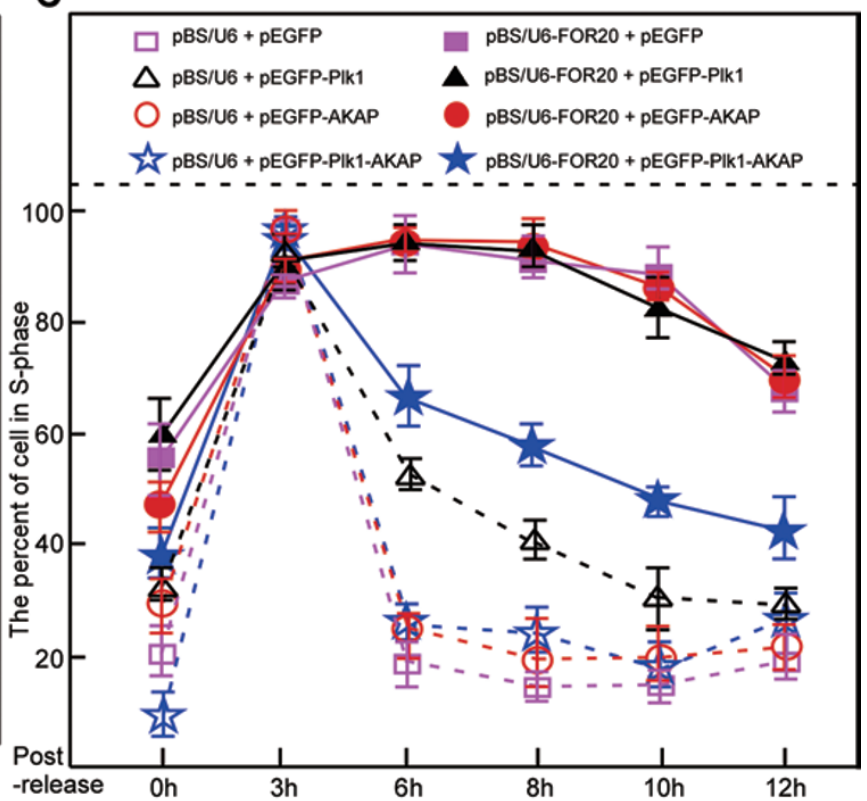

D

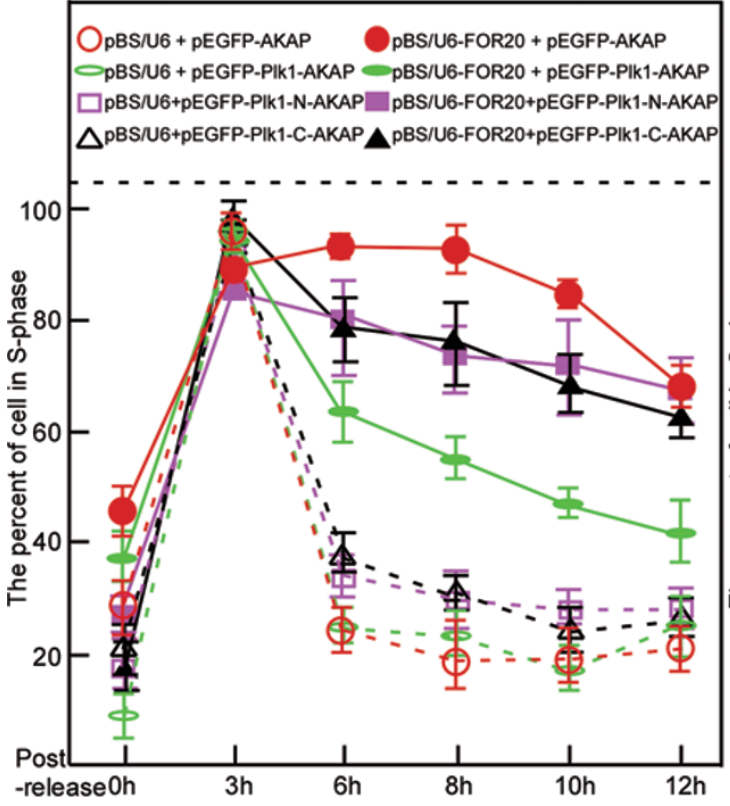

E

$\square$ pBS/U6 + pEGFP-AKAP $\quad$ pBS/U6-FOR20 + pEGFP-AKAP DBS/U6 + pEGFP-PIk1-AKAP OpBS/U6+pEGFP-PIk1-FAA-AKAP $\triangle$ pBS/U6+pEGFP-PIk1-K82M-FAA-AKAP 1 pBS/U6-FOR20+pEGFP-PIk1-K82M-FAA-AKAP

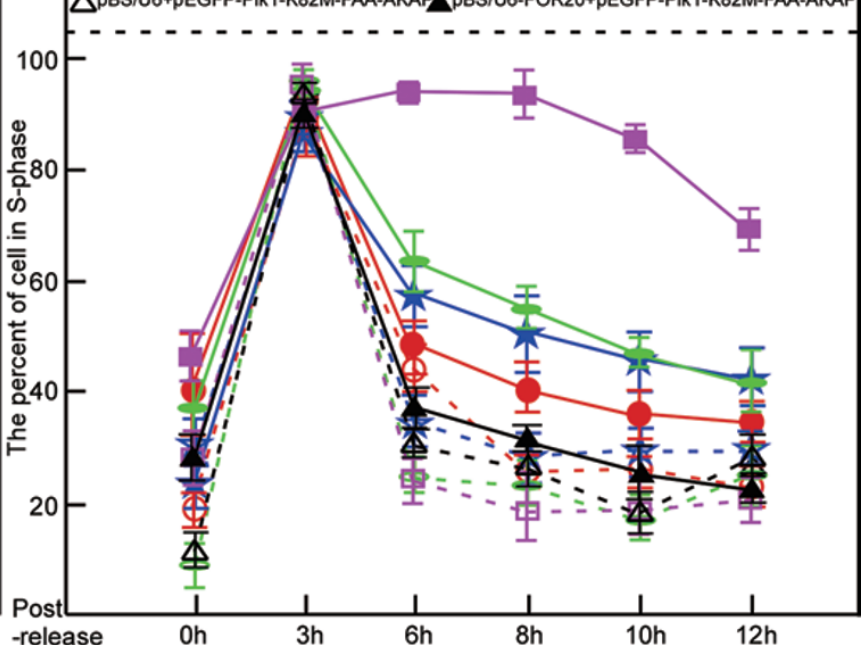


(C terminus) nor kinase domain ( $\mathrm{N}$ terminus) of Plk1 effectively reversed Plk1 depletion-induced DNA replication defects (Figure 4C and Supplementary information, Figure S8B). Interestingly, forced centrosomal targeting of kinase-dead Plk1, PBD-deficient Plk1, or even the kinase-dead, PBD-deficient Plk1, significantly reversed S-phase defects induced by FOR20 depletion (Figure 6D, 6E and Supplementary information, Figure S9BS9D). These data indicate that Plk1 may function in a yet unknown manner in centrosomes, independently of its kinase activity and canonical PBD-mediated interactions. Future studies are clearly needed to explore the underlying mechanism.

It has been demonstrated that Plk1 orchestrates a wide range of crucial events from entry into to exit from mitosis $[27,28]$. Yet, the significance of Plk1 during S-phase progression remains largely unknown. Recently, several groups have provided evidence that Plk1 may facilitate DNA replication and maintain genomic stability [16, 29, 30]. Furthermore, Plk1 promotes DNA synthesis through direct phosphorylation of Orc2 on the replication origin [16]. Our data presented here reveal that the centrosomal localization of Plk1 promotes DNA replication independently of its kinase activity, implying a previously undescribed role of centrosomal Plk1 in cell cycle regulation.

Centrosomal localization of Plk1 is required for centrosome maturation, separation and reduplication, mitotic entry and bipolar spindle formation [13, 31-33]. However, little is known about how Plk1 is targeted to centrosomes. Recent reports indicate that the centrosomal protein human cenexin 1 (hCenexin1) recruits Plk1 to centrosomes by directly interacting with the PBD of Plk1 during late G2 and M phases [34, 35]. However, a Plk1 mutant lacking the PBD is still targeted to centrosomes [19]. The PBD phosphate pincer mutant (H538A/ $\mathrm{K} 540 \mathrm{M}$ ), which fails to interact with endogenous hCenexin 1 under the condition in which it binds to phosphorylated CDC25 [35], is still localized to centrosomes in several cell lines [19]. Thus, it is likely that a yet to be identified mechanism is involved in targeting Plk1 to centrosomes. Our results show that the centrosomal protein FOR20 is required for recruiting Plk1 to centrosomes, suggesting a hitherto uncharacterized mechanism of targeting Plk1 to centrosomes.

\section{Materials and Methods}

\section{Cell culture and synchronization}

HeLa cells were maintained in Dulbecco's modified Eagle's medium (DMEM) containing $10 \%$ serum (Gibco) at $37{ }^{\circ} \mathrm{C}$ in $5 \%$ $\mathrm{CO}_{2}$. To synchronize cells, HeLa cells were treated with $2 \mathrm{mM}$ thymidine for $16 \mathrm{~h}$, released for $8 \mathrm{~h}$ and incubated with $2 \mathrm{mM}$ thymidine for another $16 \mathrm{~h}$ (double thymidine block), or treated with $2.5 \mathrm{mM}$ thymidine for $24 \mathrm{~h}$ (thymidine block) [17].

\section{Centrosome preparation}

Centrosomes were isolated from $6 \times 10^{7} \mathrm{HeLa}$ cells. After treatment with $10 \mu \mathrm{g} / \mathrm{ml}$ nocodazole and $5 \mu \mathrm{g} / \mathrm{ml}$ cytochalasin $\mathrm{B}$ for $1.5 \mathrm{~h}$, the cells were harvested and resuspended in $1 \mathrm{ml}$ of cold Tris-buffered saline- $8 \%$ sucrose buffer. $2 \mathrm{ml}$ of lysis buffer (1 mM HEPES pH 7.2, 0.5\% NP-40, $0.5 \mathrm{mM} \mathrm{MgCl}_{2}, 0.1 \%$ $\beta$-mercaptoethanol, and protease inhibitors) was added, and the cells were disrupted with 5 strokes in a glass homogenizer. The homogenate was immediately centrifuged at $2500 \times \mathrm{g}$ at $4{ }^{\circ} \mathrm{C}$ for $10 \mathrm{~min}$ to remove the swollen nuclei, chromatin aggregates and unlysed cells. The resulting supernatant was supplemented with HEPES buffer to a final concentration of $10 \mathrm{mM}$. The lysate was then placed on top of $1 \mathrm{ml}$ of $60 \%$ sucrose solution and centrifuged at $25000 \times \mathrm{g}$ at $4{ }^{\circ} \mathrm{C}$ for $30 \mathrm{~min}$. The top $2 \mathrm{ml}$ of suspension was removed to a new tube and $0.6 \mathrm{ml}$ distilled water was added. This suspension was added to the top of a discontinuous sucrose gradient (from the bottom to the top, containing 0.5, 0.4, and 0.4 $\mathrm{ml}$ of $70 \%, 50 \%$, and $40 \%$ sucrose solutions, respectively) in a 4-ml tube. After centrifugation at $96500 \times \mathrm{g}$ for $1.5 \mathrm{~h}$, the first $1 \mathrm{ml}$ from the top was removed, and 36 fractions were collected, starting from the top of the gradient, $200 \mu \mathrm{l}$ each for first 5 fractions and $60 \mu \mathrm{l}$ each for the rest of the fractions. The fractions were stored at $-80{ }^{\circ} \mathrm{C}$ after being frozen in liquid nitrogen and used for the experiments.

\section{Plasmids and transfections}

Full-length human FOR20 and Plk1 were cloned by RT-PCR and subcloned into pEGFP (pEGFP-C1; Clontech), pEYFP (pEYFP-C1), pECFP (pECFP-C1), pGST (pGEX-5X-1; Amersham Pharmacia) and pFLAG (pCMV-Tag 2C, Stratagene) vectors, respectively. pEYFP-C1 and pECFP-C1 were provided by Dr J Luo (Zhejiang University School of Medicine, China). The mutants Plk1-N (1-356 aa), Plk1-C (312-603 aa), Plk1-K82M, Plk1FAA and Plk1-FA were generated by PCR and then cloned into either $p E G F P$ or $p F L A G$ vector. The constructs of $p E G F P-A K A P$, pEGFP-Plk1-AKAP, pEGFP-Plk1-K82M-AKAP, pEGFP-Plk1FAA-AKAP, pEGFP-Plk1-FA-AKAP, pEGFP-Plk1-N-AKAP and pEGFP-Plk1-C-AKAP were generated as previously described [21]. All of these constructs were confirmed by DNA sequencing. These vectors were transfected into cells with Lipofectamine 2000

Figure 6 FOR20 influences S-phase progression by recruiting Plk1 to centrosomes. (A, B) HeLa cells treated with either pBS/U6 or pBS/U6-FOR20 for $72 \mathrm{~h}$ were transfected with pEGFP-PIk1-AKAP or pEGFP-PIk1 for another $24 \mathrm{~h}$, and were fixed and stained with antibodies against $\gamma$-tubulin (red) and FOR20 (blue). Scale bar, $10 \mu \mathrm{m}$. The percent of cells with either centrosomal GFP-PIk1-AKAP or GFP-Plk1 is shown as the mean of three independent experiments \pm SD $\left({ }^{* *} P\langle 0.01, n>\right.$ 300). (C-E) HeLa cells treated with either pBS/U6 or pBS/U6-FOR20 for $72 \mathrm{~h}$ were transfected with the indicated vectors, incubated with thymidine $(2.5 \mathrm{mM}, 24 \mathrm{~h})$, and then subjected to FACS analysis at the times shown after release. The percent of cells in S-phase is expressed as the mean of three independent experiments \pm SD. 
reagent (Invitrogen) according to the manufacturer's instructions.

\section{RNA interference}

Oligos corresponding to the following sequences were synthesized and cloned into pBS/U6 vector: 5'-AAGGGCGGCTTTGCCAAGTGCTT-3' for Plk1 RNAi (pBS/U6-Plk1) [36] and 5'-AGGTAGAGGAGAAGTAAAT-3' for FOR20 RNAi (pBS/U6-FOR20). The siRNAs sequences for Aurora A (5'-AUUCUUCCCAGCGCGUUCC-3') [37], CDK1 (5'- GGGGUUCCUAGUACUGCAA-3') [38] and control siRNA (5'-UUCUCCGAACGUGUCACGU-3') were obtained from Genepharma (GenePharma, Shanghai, China).

\section{Western blotting}

Either cell lysates or centrosome fractions were subjected to western blot analysis with anti-FOR20, $\gamma$-tubulin (Sigma), Plk1 (Sigma), cyclin B1 (Cell Signaling), cyclin A or E, GAPDH, GFP (Santa Cruz) or FLAG (Sigma) antibodies. The blots were probed with IRDye $800 \mathrm{CW}$-conjugated secondary antibodies. The IRDye $800 \mathrm{CW}$ activity was detected with the LI-COR Odyssey system (LI-COR Biosciences, USA).

\section{GST pull-down assays}

GST pull-down assays were performed as described previously [36, 39]. In brief, GST or GST-FOR20 generated in E. coli DH5 $\alpha$ was incubated with lysates of HeLa cells in TBSN buffer (20 $\mathrm{mM}$ Tris, $\mathrm{pH}$ 8.0, $150 \mathrm{mM} \mathrm{NaCl}, 0.5 \%$ NP-40, 5 mM EGTA, 1.5 mM EDTA, $0.5 \mathrm{mM} \mathrm{Na}_{3} \mathrm{VO}_{4}, 20 \mathrm{mM}$ p-nitrophenyl phosphate) supplemented with protease inhibitors at $4{ }^{\circ} \mathrm{C}$ for $2 \mathrm{~h}$. GST and GST-FOR20 were adsorbed to glutathione-agarose beads for an additional $1 \mathrm{~h}$. The bound proteins were resolved by SDS-PAGE, transferred to Immobilon-P membrane (Millipore) and subjected to western blot analysis.

\section{Flow cytometry}

For cell cycle analysis, randomly growing or synchronized cells were stained with propidium iodide and subjected to flow cytometry with the LSR II flow cytometer (BD Biosciences). FACSDiva software (BD Biosciences) was used for data acquisition and analysis. The percentage of cells in S-phase was calculated with ModFit software (BD Biosciences).

\section{Immunofluorescence}

Immunofluorescence experiments were generally performed as described previously [40, 41]. HeLa or T98G cells grown on coverslips were fixed with cold methanol $\left(-20{ }^{\circ} \mathrm{C}\right)$, stained with anti-FOR20 (home-made rabbit polyclonal antibody ), anti- $\alpha$ or $\gamma$-tubulin (Sigma-Aldrich), -cyclin A or E (Santa Cruz), -dynein, -p150 (BD Biosciences), -Plk1, -PCNA (Cell signaling), -Aurora A, -CDK1 or CDK2 (Santa Cruz) antibodies for $2 \mathrm{~h}$ at room temperature, and incubated with either Cy3-conjugated anti-mouse IgG or FITC-conjugated anti-rabbit IgG secondary antibody (Jackson ImmunoResearch) for 40 min. DNA was stained with DAPI (Sigma). Finally, the mounted coverslips were analyzed by confocal fluorescence microscopy (LSM510, Zeiss).

\section{BrdU staining}

Cells were labeled with $10 \mu \mathrm{M}$ BrdU for 20 min, washed 3 times with PBS (phosphate-buffered saline), fixed in $3.7 \%$ paraformaldehyde/PBS for $10 \mathrm{~min}$, washed 3 times with PBS, permeabilized with $0.5 \%$ Triton X-100/PBS, blocked in 5\% BSA/ PBS and stained with DAPI. After another wash with PBS, the cells were re-fixed in $3.7 \%$ paraformaldehyde for another $10 \mathrm{~min}$, extracted with $4 \mathrm{~N} \mathrm{HCl} / 1 \%$ Triton X-100 for 10 min, washed with $0.1 \% \mathrm{NP}-40 / \mathrm{PBS}$, stained with anti-BrdU ((Invitrogen) antibody for $2 \mathrm{~h}$ and incubated with either Cy3-conjugated or FITCconjugated secondary antibody for $40 \mathrm{~min}$. Finally, the mounted coverslips were analyzed by confocal fluorescence microscopy.

\section{FRET assay}

The acceptor photobleaching module of LSM510 software was used for the acceptor photobleaching FRET assay. The 458-nm laser line was used to excite CFP and the 514-nm laser line was used to excite and bleach YFP. Microscopic fields containing one or two centrosomes that expressed both CFP-Plk1 and YFP-FOR20 with similar levels of expression were selected. A region of interest was drawn on one of the centrosomes in the field or on a randomly chosen area in the cytoplasm for bleaching. FRET efficiency $(E)$ was calculated as follows: $E=($ Dpost - Dpre $) / D p o s t$ for all Dpost $>$ Dpre. Dpre and Dpost are donor fluorescence intensity before and after photobleaching, respectively [42-44].

\section{Statistical analysis}

Means and standard deviations were calculated and are shown in the graphs. Respective $n$ values are shown in the figure legends. The indicated $P$ values were obtained with two-tailed Student's $t$ tests.

\section{Acknowledgments}

We thank G Xiao for help with confocal imaging; J Cao and Y Fang for help with FACS analysis; E Erikson, Y Kang, D Li and Y Zhou for critical reading of the manuscript. This work was supported by the Ministry of Science and Technology of China (2013CB945603, 2012CB945004 and 2011CBA01001), the National Natural Science Foundation of China (31071221, 31190063 and 31125017), the 111 Project (B13026), the Natural Science Foundation of Zhejiang Province, China (Z2100247 and Y2100106), Department of Science and Technology of Zhejiang Province (2009C03012-3 and 2009F80005), Department of Health of Zhejiang Province (WKJ2006-2-014) and Zhejiang Provincial Program for the Cultivation of High-level Innovative Health talents.

\section{References}

1 Bornens M. The centrosome in cells and organisms. Science 2012; 335:422-426.

2 Nigg EA, Raff JW. Centrioles, centrosomes, and cilia in health and disease. Cell 2009; 139:663-678.

3 Doxsey S, Zimmerman W, Mikule K. Centrosome control of the cell cycle. Trends Cell Biol 2005; 15:303-311.

4 Hinchcliffe EH, Miller FJ, Cham M, Khodjakov A, Sluder G. Requirement of a centrosomal activity for cell cycle progression through G1 into S phase. Science 2001; 291:1547-1550.

5 Khodjakov A, Rieder CL. Centrosomes enhance the fidelity of cytokinesis in vertebrates and are required for cell cycle progression. J Cell Biol 2001; 153:237-242. 
6 Ferguson RL, Maller JL. Centrosomal localization of cyclin E-Cdk2 is required for initiation of DNA synthesis. Curr Biol 2010; 20:856-860.

7 Matsumoto Y, Maller JL. A centrosomal localization signal in cyclin $\mathrm{E}$ required for $\mathrm{Cdk} 2$-independent $\mathrm{S}$ phase entry. Science 2004; 306:885-888.

8 Pascreau G, Eckerdt F, Churchill ME, Maller JL. Discovery of a distinct domain in cyclin A sufficient for centrosomal localization independently of Cdk binding. Proc Natl Acad Sci USA 2010; 107:2932-2937.

9 Jackman M, Lindon C, Nigg EA, Pines J. Active cyclin B1Cdk1 first appears on centrosomes in prophase. Nat Cell Biol 2003; 5:143-148.

10 Lindqvist A, Kallstrom H, Lundgren A, Barsoum E, Rosenthal CK. Cdc25B cooperates with Cdc25A to induce mitosis but has a unique role in activating cyclin $\mathrm{B} 1-\mathrm{Cdk} 1$ at the centrosome. J Cell Biol 2005; 171:35-45.

11 Aubusson-Fleury A, Lemullois M, de-Loubresse NG, et al. FOR20, a conserved centrosomal protein, is required for assembly of the transition zone and basal body docking at the cell surface. J Cell Sci 2012; 125:4395-4404

12 Sedjaï F, Acquaviva C, Chevrier V, et al. Control of ciliogenesis by FOR20, a novel centrosome and pericentriolar satellite protein. J Cell Sci 2010; 123:2391-2401.

13 Macurek L, Lindqvist A, Lim D, et al. Polo-like kinase-1 is activated by aurora A to promote checkpoint recovery. Nature 2008; 455:119-123.

14 Barr FA, Sillje HH, Nigg EA. Polo-like kinases and the orchestration of cell division. Nat Rev Mol Cell Biol 2004; 5:429-440.

15 Zhou T, Zimmerman W, Liu X, Erikson RL. A mammalian NudC-like protein essential for dynein stability and cell viability. Proc Natl Acad Sci USA 2006; 103:9039-9044.

16 Song B, Liu XS, Davis K, Liu X. Plk1 phosphorylation of Orc2 promotes DNA replication under conditions of stress. Mol Cell Biol 2011; 31:4844-4856.

17 Li H, Wang Y, Liu X. Plk1-dependent phosphorylation regulates functions of DNA topoisomerase IIalpha in cell cycle progression. J Biol Chem 2008; 283:6209-6221.

18 Yim H, Erikson RL. Polo-like kinase 1 depletion induces DNA damage in early $\mathrm{S}$ prior to caspase activation. Mol Cell Biol 2009; 29:2609-2621.

19 Garcia-Alvarez B, de Carcer G, Ibanez S, Bragado-Nilsson E, Montoya G. Molecular and structural basis of polo-like kinase 1 substrate recognition: Implications in centrosomal localization. Proc Natl Acad Sci USA 2007; 104:3107-3112.

20 Ji JH, Jang YJ. Screening of domain-specific target proteins of polo-like kinase 1: construction and application of centrosome/kinetochore-specific targeting peptide. $J$ Biochem Mol Biol 2006; 39:709-716.

21 Kishi K, van Vugt MA, Okamoto K, Hayashi Y, Yaffe MB. Functional dynamics of Polo-like kinase 1 at the centrosome. Mol Cell Biol 2009; 29:3134-3150.

22 Ferguson RL, Maller JL. Cyclin E-dependent localization of MCM5 regulates centrosome duplication. J Cell Sci 2008; 121:3224-3232.

23 Ferguson RL, Pascreau G, Maller JL. The cyclin A centrosomal localization sequence recruits MCM5 and Orc1 to regulate centrosome reduplication. J Cell Sci 2010; 123:2743-
2749.

24 Knockleby J, Lee H. Same partners, different dance: involvement of DNA replication proteins in centrosome regulation. Cell cycle 2010; 9:4487-4491.

25 Stuermer A, Hoehn K, Faul T, et al. Mouse pre-replicative complex proteins colocalise and interact with the centrosome. Eur J Cell Biol 2007; 86:37-50.

26 Tsvetkov L, Stern DF. Interaction of chromatin-associated Plk1 and Mcm7. J Biol Chem 2005; 280:11943-11947.

27 Petronczki M, Lenart P, Peters JM. Polo on the rise-from mitotic entry to cytokinesis with Plk1. Dev Cell 2008; 14:646659.

28 Strebhardt, K. Multifaceted polo-like kinases: drug targets and antitargets for cancer therapy. Nat Rev Drug Discov 2010; 9:643-660.

29 Lei M, Erikson RL. Plk1 depletion in nontransformed diploid cells activates the DNA-damage checkpoint. Oncogene 2008; 27:3935-3943.

30 Trenz K, Errico A, Costanzo V. Plx1 is required for chromosomal DNA replication under stressful conditions. EMBO J 2008; 27:876-885.

31 Archambault V, Glover DM. Polo-like kinases: conservation and divergence in their functions and regulation. Nat Rev Mol Cell Biol 2009; 10:265-275.

32 Liu X, Erikson RL. Activation of Cdc2/cyclin B and inhibition of centrosome amplification in cells depleted of Plk1 by siRNA. Proc Natl Acad Sci USA 2002; 99:8672-8676.

33 Loncarek J, Hergert P, Khodjakov A. Centriole reduplication during prolonged interphase requires procentriole maturation governed by Plk1. Curr Biol 2010; 20:1277-1282.

34 Soung NK, Park JE, Yu LR, et al. Plk1-dependent and -independent roles of an ODF2 splice variant, hCenexin1, at the centrosome of somatic cells. Dev Cell 2009; 16:539-550.

35 Soung NK, Kang YH, Kim K, et al. Requirement of hCenexin for proper mitotic functions of polo-like kinase 1 at the centrosomes. Mol Cell Biol 2006; 26:8316-8335.

36 Zhou T, Aumais JP, Liu X, Yu-Lee LY, Erikson RL. A role for Plk1 phosphorylation of NudC in cytokinesis. Dev Cell 2003; 5:127-138.

37 Hirota T, Kunitoku N, Sasayama T, et al. Aurora-A and an interacting activator, the LIM protein Ajuba, are required for mitotic commitment in human cells. Cell 2003; 114:585-598.

38 Johnson N, Li YC, Walton ZE, et al. Compromised CDK1 activity sensitizes BRCA-proficient cancers to PARP inhibition. Nat Med 2011; 17:875-882.

39 Zhu XJ, Liu X, Jin Q, Cai Y, Yang Y, Zhou T. The L279P mutation of nuclear distribution gene $\mathrm{C}$ (NudC) influences its chaperone activity and lissencephaly protein 1 (LIS1) stability. J Biol Chem 2010; 285:29903-29910.

40 Yang Y, Yan X, Cai Y, Lu Y, Si J, Zhou T. NudC-like protein 2 regulates the LIS1/dynein pathway by stabilizing LIS1 with Hsp90. Proc Natl Acad Sci USA 2010; 107:3499-3504.

41 Cai Y, Yang Y, Shen M, Zhou T. Inhibition of cytokinesis by overexpression of NudCL that is localized to the centrosome and midbody. Cell Res 2009; 19:1305-1308.

42 Breunig M, Lungwitz U, Liebl R, Goepferich A. Fluorescence resonance energy transfer: evaluation of the intracellular stability of polyplexes. Eur J Pharm Biopharm 2006; 63:156165 . 
43 Douglas C, Youvan-William JC, Chris M, et al. Fluorescence imaging micro-spectrophotometer (FIMS). Biotechnol et alia 1997; 1:1-16.

44 Gordon GW, Berry G, Liang XH, Levine B, Herman B. Quan- titative fluorescence resonance energy transfer measurements using fluorescence microscopy. Biophys $J$ 1998; 74:27022713.

(Supplementary information is linked to the online version of the paper on the Cell Research website.) 